\title{
$2 \mathrm{~d}$ quantum dilaton gravity as/versus finite dimensional quantum mechanical systems
}

\author{
T. Strobl ${ }^{\mathrm{a}}$ \\ ${ }^{a}$ Institut für Theoretische Physik E, RWTH-Aachen, \\ Sommerfeldstr. 26-28, D-52056 Aachen, Germany
}

In this talk we will deal with quantum aspects of generalized $2 \mathrm{~d}$ dilaton theories. The classical features of these models were discussed already in the talk by Thomas Klösch [1]. We will also refer to [1] for the Lagrangian, Eq. ([1].1), which governs the dynamics for our $2 \mathrm{~d}$ metric $g$, the scalar dilaton field $\Phi$, and, if $K \neq \equiv 0$, the Yang-Mills connection $A=A^{Y M}$. It contains four functions $U, V, W, K$ to be fixed so as to define the system, allowing for a simultaneous treatment of a whole family of models.

My talk will have three parts: First, I will summarize what one might call the "Chern-Simons"formulation of generalized $2 \mathrm{~d}$ dilaton gravity [2, 3]. Second, I will deal with the Hamiltonian quantization of these models [3, 4 . As one of the results of this analysis we will obtain the spectrum of the mass operator, finding it to be sensitive to the signature of the theory as well as to the choice of functions $U, V, W, K$ in (近.1). Third, I will make some remarks on the statistical mechanical entropy that one obtains for the $2 \mathrm{~d}$ models [5] when applying the approach of Carlip as well as Balachandran, Chandar and Momen [6].

Although we deal with a two-dimensional field theory, on all of the above three occasions one finds finite dimensional particle systems to play a central role. Before going into further details in separate sections below, I intend to outline the main idea of how these relations come about:

1) Given a Lagrangian ([1].1) with, for presentational reasons, $K \equiv 0$ ( $\Rightarrow$ no Yang-Mills fields) we will associate to it an auxiliary $\mathbb{R}^{3}$ with coordinates $X^{i}, i=1,2,3$, that is equipped with a Poisson bracket $\left\{X^{i}, X^{j}\right\}=\mathcal{P}^{i j}(X)$. Here the $\left(X^{i_{-}}\right.$ dependent) matrix $\mathcal{P}^{i j}$ is determined uniquely by the three "potentials" $U, V, W$. For dimensional reasons $\mathbb{R}^{3}$ cannot be symplectic. However, the $\mathbb{R}^{3}$ foliates (stratifies) into two-dimensional submanifolds which are symplectic. Correspondingly, these "symplectic leaves" may be regarded as phase spaces of fictitious point particles (although in general these phase spaces will have non-trivial topology and not be a cotangent bundle). It turns out that this $\mathbb{R}^{3}$ equipped with the Poisson bracket $\mathcal{P}^{i j}$ can serve as a target space of a $2 \mathrm{~d} \sigma$-model which is equivalent to the original theory described by ([1].1), while providing powerful techniques not available in the metrical formulation.

2) Like any gravitational system the Lagrangian

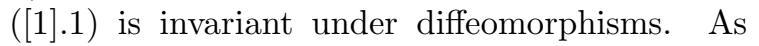
demonstrated in the previous talk 11 (and references therein), for a given topology of the spacetime manifold $\mathcal{M}$ the space of solutions to the field equations modulo diffeomorphisms is finite dimensional only. This is characteristic for a topological field theory. For $\mathcal{M} \sim S^{1} \times \mathbb{R}$ and $K \equiv 0$, in particular, the solution space was found to be two-dimensional, one of the two parameters being the "mass" $M$ of the spacetime. In a Hamiltonian treatment of the model ([1].1) a symplectic reduction leads to a phase space that is equivalent to this solution space. Correspondingly we are again left with a two-dimensional symplectic manifold (orbifold), which this time is the reduced (i.e. physical) phase space RPS of the dilaton gravity theory. This makes the $2 \mathrm{~d}$ theory appear as a point particle model. However, in general the RPS has a non-trivial topology. Similarly, in a Dirac quantization the only continuous parameter the physical wave functions $\Psi_{p h}$ 
depend on is found to be $M$ (in an appropriate polarization). But again, in general there are also further discrete labels $m, l, \Psi_{p h}=\Psi_{p h}(M, m, l)$, which are intertwined with $M$ in a nasty manner. While this shall be made more transparent in the Sec. 2 below, let me mention here only that the discrete labels are a relic of the higher dimension where the theory was defined before the reduction. In those cases where they are present, they are the obstacle of regarding the $2 \mathrm{~d}$ system to be identical to a standard point particle system on a line.

3) Carlip and Balachandran et al. regard spacetimes $\mathcal{M}$ with boundary $\partial \mathcal{M}$. Identifying $\partial \mathcal{M}$ with the (stretched) horizon of a black hole, its entropy is suggested to stem from tracing over quantized boundary modes. At the example of $2+1$ gravity in its Chern-Simons formulation Carlip developed a recipe of how to induce from the bulk action on $\mathcal{M}$ an action that governs the dynamics of the boundary modes on $\partial \mathcal{M}$. Given our formulation of ([1].1), we will be able to adapt this recipe to the general class of $1+1$ gravity theories. Note that since now $\mathcal{M}$ is $1+1$ dimensional, its boundary will be $0+1$ dimensional, i.e. here the boundary modes are point particles. They are distinct from the bulk degrees of freedom certainly and thus have to be distinguished from the finite dimensional modes encountered in the two items above. Still, we will find a very nice relation to the fictitious particles on the target space; in fact, in a certain sense, the latter become alive and real by the approach of [6].

\section{1. "Chern-Simons" formulation of gener- alized 2d dilaton gravity}

One of the major steps in the realm of $2+1$ gravity was its reformulation in terms of a ChernSimons gauge theory,

$$
\int d^{3} x \sqrt{|g|}(R+\Lambda) \leftrightarrow \int \operatorname{tr}\left(A d A+\frac{2}{3} A^{3}\right) .
$$

Here the one-forms $A_{i}, i$ running over the Lie algebra of the gauge group, have been identified with the vielbein and the spin connection of an Einstein-Cartan formulation of the gravity theory. A similarly powerful reformulation of the general class of gravity models ([1].1) exists in terms of Poisson $\sigma$-models [2, 3]:

$L\left[g, \Phi, A^{Y M}\right] \leftrightarrow \int_{\mathcal{M}} A_{i} \wedge d X^{i}+\frac{1}{2} \mathcal{P}^{i j} A_{i} \wedge A_{j}$

We comment on this relation for the case that $K=W=0, U=\Phi, V$ arbitrary, differentiable: Then $i, j=1,2,3$, again the $A$ 's are zweibein and spin connection, $A_{i}=\left(e^{1}, e^{2}, \omega^{1}{ }_{2}\right)$, while $X^{3} \equiv \Phi$, and $X^{1}$ and $X^{2}$ have been introduced to ensure zero torsion in an Einstein-Cartan formulation. The antisymmetric $3 \times 3$-matrix $\mathcal{P}$ is defined by $\mathcal{P}^{12}=V\left(X^{3}\right), \mathcal{P}^{13}=-X^{2}$, and $\mathcal{P}^{23}= \pm X^{1}$, where the two signs correspond to Euclidean and Lorentzian signature of the gravity theory, respectively. Two decisive observations: 1) Regarding $X^{i}$ as coordinates in an $\mathbb{R}^{3}$ (target space) and the lower index $i$ at $A_{i} \equiv$ $A_{i \mu} d x^{\mu}$ as a one-form index on this space, the action (2) is covariant with respect to "coordinate changes" $X^{i} \rightarrow \widetilde{X}^{i}(X)$. 2) The matrix $\mathcal{P}$ satisfies $\mathcal{P}^{i l} \partial \mathcal{P}^{j k} / \partial X^{l}+$ cycl. $(i, j, k)=0$ so that indeed $\left\{X^{i}, X^{j}\right\}:=\mathcal{P}^{i j}$ defines a Poisson bracket.

The main technical advantage of the new formulation consists in the existence of the following powerful tools: Locally the Poisson bracket (in the target space) allows for Casimir-Darboux coordinates. In the above example they may be chosen as $\widetilde{X}^{i}:=\left(M, \varphi, X^{3}\right)$ with

$M= \pm\left(X^{1}\right)^{2}+\left(X^{2}\right)^{2}+2 \int^{X^{3}} V(u) d u$,

while $\varphi=\arctan \left(X^{2} / X^{1}\right)$ and $\varphi=\ln \left(X^{1}+X^{2}\right)$ for Euclidean and Lorentzian signature, respectively. Due to the simplification of the Poisson tensor in these coordinates the r.h.s. of (2) trivializes locally to $\int A_{\tilde{i}} \wedge d \widetilde{X}^{i}+A_{\tilde{2}} \wedge A_{\tilde{3}}$ where $A_{\tilde{i}} \equiv A_{j} \partial X^{j} / \partial \widetilde{X}^{i}$. Global information is restored by taking into account the topology of the symplectic leaves, which coincide with the connected components of the level surfaces of the Casimir function (3). (1)

These tools facilitate even the analysis for a $\mathcal{P}^{i j}$ linear in $X$, in which case (2) takes the form of an ordinary $B F$-gauge theory (cf., e.g., [3, ], ).

\footnotetext{
${ }^{1}$ At values of $M$ marking a transition in topology of the leaves, the connected components of the level surfaces $M=$ const. may still consist of several symplectic leaves.
} 


\section{Hamiltonian quantization}

The r.h.s. of (2) is already in first order form, so its Hamiltonian structure is determined readily. Denoting the spacetime coordinates with $r, t$, where $r$ may be a coordinate on either $S^{1}$ or $\mathbb{R}, X^{i}(r)$ becomes conjugate to $A_{j r}(\bar{r})$, i.e. $\left\{X^{i}(r), A_{j r}(\bar{r})\right\}=\delta_{j}^{i} \delta(r-\bar{r})$, while the $A_{j t}(r)$ become Lagrange multipliers for the first class constraints $G^{i}(r) \equiv X^{i^{\prime}}+\mathcal{P}^{i j}(X) A_{j r} \approx 0$. Clearly the field theoretic Poisson bracket has nothing to do with the one on the target space and thus should be distinguished sharply from it.

\subsection{Reduced phase space}

Here we have to look at the quotient $\operatorname{space}\left(G^{i}(r) \approx 0\right) /\left\{G^{i}(r), \cdot\right\}=$ : RPS. Taking into account boundary conditions, RPS coincides topologically with the space of solutions to the field equations modulo diffeomorphisms (if one excludes incomplete spacetimes with kinks, to be precise). Thus in the case of periodic boundary conditions in $r$ a direct comparison with the solution space for $\mathcal{M}=S^{1} \times \mathbb{R}$, summarized in [1, is accessible. Indeed this solution space was found to be two-dimensional, one parameter being a "mass"-parameter $M$, which in fact coincides with the (constant) value of (3) on $\mathcal{M}$, while the other one, which we shall call $P$ here, was given a geometrical interpretation at the beginning of Sec. 3.1 and in Sec. 3.3 of [1]. It may be shown that $P=\oint A_{\widetilde{1}} d r$. Correspondingly the induced Poisson bracket on the RPS may be calculated, yielding $\{M, P\}=1$. In the simply connected case $\mathcal{M}=\mathbb{R}^{2}$, on the other hand, the solution space was found to be onedimensional in [1.2], being parametrized by $M$. Here $P=\int_{r_{\min }}^{r_{\max }} A_{\widetilde{1}} d r$ becomes a second gaugeinvariant parameter upon appropriate choices of boundary conditions at $r=r_{\min , \max }$; it then yields the asymptotic Killing time difference of the $(t=0)$-hypersurface $[8]$. So, in any case the RPS is two-dimensional. However, generically its topology is quite non-trivial. Depending on the choice of the potentials $U, V, W, K$, one may run into qualitatively different situations:

Simpler cases: This occurs for those potentials where the integer $n$ defined in Sec. 1.2 of [i] is at most one, or, equivalently, whenever all the symplectic leaves in the target space are simply connected. Spherically symmetric $4 \mathrm{~d}$ gravity as well as string inspired gravity belong to this class of models. Here RPS $=\mathbb{R}^{2}$ with globally conjugate variables $M \in \mathbb{R}$ and $P \in \mathbb{R}$. Thus the RPS is equivalent to the one of a point particle on the line. Correspondingly, up to unitary equivalence, $\Psi=\Psi(M), P \rightarrow-i \hbar d / d M$, and $\Psi \in \mathcal{H}=\mathcal{L}^{2}(\mathbb{R}, d M)$.

Generic cases: Whenever $n_{\max } \geq 2$, which occurs, e.g., whenever $V$ is not positive or negative definite in the example $W=K=0, U=\Phi$ above. Here the topology of RPS is non-trivial, since along the range of $M$ the value of the integer $n$ will change. A typical scenario: For $M<M_{1}$ and $M>M_{2}$ one has $n=0$ with a completely homogenous (or stationary) Penrosediagram (cf. Fig. 1 in [四); besides $M$ these solutions are labelled by one further continuous parameter $P \in \mathbb{R}$. For $M_{1}<M<M_{2}$, however, $n=2$ and beside $P \in \mathbb{R}$ there is an additional discrete label $l$ of the solutions, the integer patch number in the fundamental region, cf. Fig. 5 of [1]. Thus now $\Psi=\Psi(M, l)$, but $l$ may be nonzero only within a certain range of $M$. Correspondingly, we do not know the inner product for these wave functions yet and quantization seems ambiguous in these cases.

The above analysis was valid for Lorentzian signature. For Euclidean signature there are choices of the potentials such that $P$ takes values from a finite interval only. In such cases the spectrum of the conjugate variable $M$ will be discrete.

\subsection{Dirac quantization}

We have to solve the functional equations

$$
\left[X^{i^{\prime}}(r)+i \hbar \mathcal{P}^{i j}(X(r)) \frac{\delta}{\delta X^{j}(r)}\right] \Psi[X(r)]=0 .
$$

Given the above factor ordering, there are no anomalies and thus (1) is integrable locally. In the case of our three-dimensional target space with the Poisson tensor defined by the single function $V$, the local solution to (仼) has the form: $\Psi[X(r)]=\delta\left[M^{\prime}(r)\right] \exp ((i / \hbar) \oint \alpha) \Psi_{0}(M)$. Here $M$ is the functional defined in (3) and the first factor yields a restriction to maps $X(r)$ lying 
entirely in a symplectic leaf $M=$ const. Furthermore, $d \alpha=\Omega$ where $\Omega$ is the symplectic form on the respective leaf, induced by the target space Poisson bracket. As a result of the analysis [3] for a global solution to the quantum constraints, we merely have to determine the topology of the level surfaces (3). Let $r \sim r+2 \pi$ (periodic boundary conditions). The topological invariants of relevance are $\pi_{0}(M=$ const $)$, $\pi_{1}(M=$ const $)$, and $\pi_{2}(M=$ const $) . \quad \pi_{0} \neq 0$ and/or $\pi_{1} \neq 0$ lead to an additional discrete label in $\Psi_{0}: \Psi_{0} \rightarrow \Psi_{0}(M, m, l), m \in \pi_{0}, l \in \pi_{1}$. While in the Lorentzian case $m$ may often be disposed of by the discrete Lorentz symmetry $X^{1} \leftrightarrow-X^{1}$, the integer $l$ is precisely the quantity encountered in [1] and the previous subsection as patch number. Here it is the winding number of the argument loop of $\Psi$ around non-simply connected pieces of the leaf $M=$ const. Since the topology of the leaves will in general change with the value of $M$, we again get this entanglement of $l$ with $M$. In our present context $\pi_{2}(M=$ const $) \neq 0$ iff (3) is compact. For an $M$ with a compact surface (3), there exists a physical wave function only if

$\oint \Omega=2 \pi n \hbar \quad, \quad n \in \mathbb{Z}$,

where the integral is taken over all of the leaf. Note that in general this may yield a discrete spectrum for $M$. Be warned also not to confuse $\Omega$ with the symplectic form of the field theory; it is the one on the leaf $M=$ const. in the target space. The condition (5) arises as an additional global integrability condition to the quantum constraints (4). As a general fact, for the Lorentzian signature $\pi_{2}$ (leaves) $=0$, while for the Euclidean signature $\pi_{1}$ (leaves) $=0$. Irrespective of the signature, in the simpler cases (defined as in Sec. 2.1) $\pi_{1}=\pi_{2}=0$ and we again obtain $\Psi_{\text {phys }} \leftrightarrow \Psi_{0}(M)$. A first example with nontrivial $\pi$ 's is provided by $V(\Phi)=\Phi(2 \mathrm{~d}$ deSitter gravity): In the Lorentzian theory the leaves (3) are not simply connected for $M>0$, while they are for $M<0$. In the Euclidean theory $\pi_{2}=\mathbb{Z}$ and (5) leads to $M=n^{2} \hbar^{2} / 4 ;|n\rangle$ gives a basis for the physical wave functions in this case. Generically, however, the spectrum of $M$ is mostly continuous also in the Euclidean case 3,4, 4].

\section{Statistical mechanical entropy}

Locally a classical solution of our gravity system (with $K \equiv 0$ ) is determined by the value of $M$, which is a constant over spacetime. Now, given a classical solution $\mathcal{M}$ with boundary $\partial \mathcal{M}$ and mass $M$, Carlip's recipe may be applied to obtain the boundary action and from it the phase space of the boundary particles. As a result of the analysis [5] one finds that this edge particle phase space is isomorphic to the symplectic leaf singled out from the Poisson bracket in the target space by the value of $M$ and Eq. (3). Applying the method of geometric quantization to this phase space of the edge modes, we obtain Eq. (5) as a consistency condition. Note that the bulk modes are left unquantized in this approach; still, by quantizing the analogue of Carlip's boundary modes, one obtains precisely the same spectrum of $M$ as in a Dirac quantization of the bulk modes. The attempt to determine the entropy of the respective black hole by a simple counting of physical "boundary states" may be successful only in the case of a compact phase space. Only in that case the Hilbert space of the boundary modes is finite dimensional, and, according to (5), its dimension may be approximated very well by the integer $n$. The result, $S \sim \ln n$, gives, however, only about the logarithm of what one expects for the entropy $S$ from other, semiclassical considerations. For most choices of $U, V, W$, however, the symplectic leaves are non-compact in the Lorentzian and the Euclidean theory, and a simple counting like this does not make sense.

\section{REFERENCES}

1. T. Klösch, this volume or gr-qc/9701012.

2. T. Klösch and T. Strobl, Class. Quantum Grav. 13 (1996) 965.

3. P. Schaller and T. Strobl, Mod. Phys. Letts. A9 (1994), 3129; LNP 469 (Springer 1996), 321 or hep-th/9507020; and Refs. therein.

4. T. Klösch and T. Strobl, in preparation.

5. J. Gegenberg, G. Kunstatter, and T. Strobl, gr-qc/9612033.

6. S. Carlip, this volume and Refs. therein.

7. R. Jackiw, this volume and Refs. therein.

8. K. Kuchar, this volume and Refs. therein. 